\title{
ESTRATEGIA DE FORMACIÓN PERMANENTE DEL MAESTRO PRIMARIO PARA LA MEDIACIÓN EN CONFLICTOS
}

\author{
MSc. Yamila del Carmen Camacho Sojo \\ Universidad de Oriente \\ yamila.camacho@uo.edu.cu
}

Palabras claves: Estrategia, mediación, conflictos.

Keywords: Strategy, mediation, conflicts
Recepción: 8 de marzo 2016

Aceptación: 26 de marzo de 2016

\section{RESUMIEN}

El presente trabajo ofrece fundamentos teóricos y prácticos sobre la utilidad de estrategias para la apropiación del método de mediación en conflictos escolares desde la formación permanente del maestro primario, como una vía imprescindible para actualizar los recursos humanos en educación y elevar el desempaño en la labor educativa del mismo. Se emplearon métodos como la entrevista, encuesta, el análisis documental, entre otros y se obtienen resultados favorables para la comprensión y aplicación del método de mediación educativa en la escuela primaria. Por lo que consideramos de gran significación práctica su aplicación en el contexto cubano.

\section{ABSTRACT}

The present work offers theoretical and practical basics about the utility of strategies for the appropriation of the mediation method in school conflicts from the primary teacher's permanent formation, like an indispensable road to upgrade the human resources in education and to elevate the desempaño in the educational work of the same one. Methods like the interview were used, you interview, the documental analysis, among other and favorable results are obtained for the understanding and application of the method of educational mediation in the primary school. For what we consider of practical great significance your application in the Cuban context. 


\section{INTRODUCCIÓN}

Constituye una prioridad del sistema de educación cubano, el perfeccionamiento de la formación permanente de los profesionales de la educación, que se concreta en los fines y objetivos de cada nivel educativo, de manera que se logre una acción transformadora y consciente en la formación integral en la personalidad de los escolares, en tanto implica comprender que la formación del maestro se extiende a lo largo de su vida profesional respondiendo a las necesidades personales, prioridades y reclamos sociales que estimulan la adquisición, actualización y perfeccionamiento de los conocimientos, habilidades y actitudes inherentes a su labor profesional, a partir de concebir la escuela como el escenario esencial que orienta y guía la formación de la personalidad de los escolares.

En el ámbito internacional las concepciones de J. A. Comenius, L. Klingberg, (1954, 1962, 1972); A. S. Makarenko, (1956); M. Danilov, (1963, 1969); constituyeron una valiosa contribución al estudio del proceso de formación de la personalidad reconociendo el papel educativo de la enseñanza; la unidad de la educación, la enseñanza y la formación como procesos que se intercondicionan y apoyan entre sí, unido a la habilidad de ver en cada educando fuerzas positivas.

A nivel nacional se han realizado numerosas investigaciones científicas que ofrecen fundamentos teóricos y metodológicos sobre la superación, capacitación o formación continua y permanente del profesional de la educación, autores como O. Castro (1997), Valcárcel, N (1998); Pérez La O. (1990), R. Forneiro (1996), articulan aspectos relacionados con la superación de los profesionales. Se destaca la doctora J Añorga desde (1986,1994, 1995,1998 y 2001), que ha sido la que más ha sistematizado lo referente a la educación de avanzada dirigida a fundamentar los procesos de superación y/o capacitación de los recursos humanos profesionales y de la comunidad como el objeto de estudio, principios generales y regularidades que permiten conformar el subsistema educativo de la Educación Avanzada, aportó el sistema de principios que norman el proceso de organización y desarrollo de la superación de los recursos humanos, conceptos, métodos, formas y tecnologías que utiliza la Educación Avanzada, para el mejoramiento de los recursos laborales y humanos.

González, D (2001), propone un proyecto de superación para maestros primarios sobre Educación Avanzada relacionado con la formulación de problemas matemáticos, Pérez, A. M. (2002); Ugalde, L. (2003); Santisteban, M. L. y C. Gato (2003) manejan en sus estudios alternativas para diseñar la formación permanente de maestros en temas específicos propios de necesidades de especialización; de igual manera V. Arencibia y G. Hernández (2003) investigaron sobre la formación continua del profesional de la educación; M. C. Roja (2009) sobre la auto superación de profesores a tiempo parcial de la Educación Primaria. R. Venet (2009) desarrolló una investigación sobre la formación permanente del profesional de la educación en atención a la diversidad, a partir de un estudio general en el nivel primario, todo lo contribuye a elevar la preparación del profesional de la educación.

Sin embargo ha sido insuficientemente abordado y poco sistematizado las particularidades esenciales para la apropiación del método de mediación educativa en los conflictos escolares, desde la formación 
permanente del maestro primario, donde en la revisión de documento y diagnóstico inicial de esta investigación(encuestas y entrevista a maestros) se reconoce que en la escuela primaria emergen dificultades en las relaciones interpersonales entre los escolares, devenidas en conflictos, por la limitación de los maestros para incorporar estilos de relaciones que favorezcan los procesos de aproximación interpersonal, como resultado de la apropiación de patrones o estereotipos poco efectivos que prevalecen en los diversos contextos sociales en los que se involucran los escolares y que trasciende hacia sus propios procesos de desarrollo personal y social.

Las referidas dificultades se manifiestan en no acatamiento de normas, incumplimiento de los deberes escolares, riñas, comportamientos inadecuados dentro y fuera del centro, falta de respeto, agresividad, malas relaciones de convivencia, etc. En este sentido, es importante significar la necesidad de que el proceso formativo como proceso pedagógico que se asocia a la apropiación de saberes, conductas y actuaciones en un contexto sociocultural determinado, sea enfocado con una nueva visión educativa, que implica brindar especial atención a la mediación en conflictos ,a la prevención educativa.

En consecuencia el tema conflicto ha sido objeto de investigación de psicólogos, pedagogos y otros profesionales de la educación en general de diferentes países que han brindado aportes teóricos y prácticos, a partir de sus realidades educativas, se destacan trabajos de Deutsch, M (1969); Schnuck y Schnuck, (1983); Ovejero (1989); Marina y Xavier Gimero, Soria (1993); Álvarez Fernández, Manuel (1993); Moore, ch (1994); Cortina, Adela (1997); Fuñes Lapponi y Saint Mezard, Damián (2001); Martínez- Otero (2001), Beltrán (2002);Remo Entelman (2002); Picard Chery, I (2002); Tomás Folch, José, Antonio (2007),entre otros. Cervantes Loredo María Teresa (2002); estrategia educativa para la solución de conflictos interpersonales en las organizaciones políticas de Nuevo León México, entre otros.

Todos estos autores de una manera $\mathrm{u}$ otra han dirigido sus análisis al incremento de los conflictos escolares por la presencia de la violencia escolar; por la inexistencia de mecanismos alternativos para la resolución de conflictos escolares; el desafío de sensibilizaciones de los agentes socializadores y la necesidad de comunicarse a través del diálogo; por la transgresión a las normas institucionales, por el aumento de expedientes disciplinarios y sanciones por conductas violentas, vandálicas o disruptivas, insultos, amenazas, rechazo, marginación, entre otras.

Las investigaciones recientes en Cuba, destacan los trabajos de Fuentes Ávila, Mara (2000) y Pérez Yera, Armando y Ortiz Torres, Emilio (2001); que utilizan la técnica del entrenamiento socio psicológico para la mediación de situaciones de conflictos entre un grupo de adolescentes de ciudad de la Habana. (2005) Yuliet Cruz Martínez, Belkis Cabrera de los Santos Finalé y Daybel Pañellas Álvarez, que implementaron un programa de mediación con niños de edad escolar en una escuela primaria de ciudad de la Habana con logros positivos. Parada Ulloa, Adaris (2007)

El método de situación de conflicto para la formación de actitudes ambientales en estudiantes de Secundaria Básica de Santiago de Cuba. Otros trabajos sobre mediación en el aprendizaje y en el uso 
de las TIC, así como la mediación educativa interdisciplinar para la elaboración de proyectos de vida profesional de los estudiantes de Ciencias Pedagógicas de Santiago de Cuba de Mayet Wilson, Mirta (2012). Sin embargo no se recoge resultado alguno de investigaciones que tracen estrategias o modelos educativos que permita desde la formación permanente del profesional de la educación primaria diseñar acciones para apropiarse del método de la mediación educativa en la prevención de conflictos escolares.

En tal sentido se realizó el análisis del Plan D de la carrera Educación Primaria, para identificar el tratamiento al método de mediación educativa y los fundamentos de la teoría de conflictos, valorando que en las asignaturas del plan de estudio se trabajan los métodos educativos para la formación en valores y el desarrollo de la personalidad de los educandos, sin embargo no incluye en su análisis el tratamiento del método de la mediación educativa en conflictos escolares.

Además se realizó el análisis de los planes de superación de los maestros primarios del último quinquenio, comprobando que ha estado dirigido a contribuir desde la formación permanente a elevar el desempeño del maestro en el tratamiento metodológico de los contenidos fundamentales de las asignaturas priorizados, a enriquecer el trabajo de las adecuaciones curriculares, las líneas que se declaran benefician el tratamiento a la didáctica de la escuela primaria, al idioma ingles e español, a la dirección científica, al maestro tutor y la labor educativa desde los proyectos educativos, a enriquecer el trabajo con los valores, evidenciándose un desequilibrio en la planificación, control y ejecución de los planes de superación desde el nivel provincial, municipal y de centro, en tanto se prioriza los contenidos de las asignaturas priorizadas y no se prioriza la atención a cómo se manifiestan los conflictos escolares, cómo se analizan o resuelven, a determinar la eficacia en los métodos educativos y a cómo realizar trabajo preventivo para mejorar la convivencia escolar y los ambientes educativos.

La situación resulta de mayor complejidad al reconocer que no obstante los esfuerzos desarrollados y los cambios que han sido introducidos en el accionar de la escuela primaria, en el diagnóstico factico realizado en la práctica educativa se han constatado insuficiencias en la dirección del proceso educativo, evidenciadas en:

Limitados recursos psicológicos para determinar las manifestaciones, causas y factores que condicionan la ocurrencia de conflictos escolares.

Existencia de inadecuados estilos de comunicación que afectan las relaciones interpersonales, la organización escolar y la convivencia en el contexto educativo.

$\checkmark$ Limitado vínculo en la relación escuela, familia y comunidad para el desarrollo de acciones educativas.

4. Las variantes de superación del maestro primario está centralizada en la preparación metodológica de cada ciclo, o nivel y ninguna otra forma.

$\checkmark$ El contenido de la formación permanente está dirigida esencialmente al tratamiento metodológico de los contenidos fundamentales de las asignaturas priorizadas y no distingue la preparación para el desarrollo de la labor educativa. 
Insuficiente dominio y utilización de métodos educativos para el tratamiento a los conflictos escolares.

Por lo que reflexionamos en los resultados parciales de la investigación referida a la elaboración de una estrategia pedagógica de superación para la formación permanente del maestro primario para la mediación educativa en conflictos escolares.

\section{DESARROLLO}

La formación permanente del maestro primario, en medio del perfeccionamiento del modelo de escuela primaria actual en el país exige cambios en la formación inicial y posgraduada, la necesidad de potenciar la formación permanente del docente, hacia la atención diferenciada a los problemas de la formación de los escolares y a las contradicciones sociales que se presentan en el proceso educativo en general. En consecuencia con esta premisa, la formación permanente del maestro primario obliga a la búsqueda de una nueva visión en el proceso de renovación y actualización de los métodos educativos, procedimientos, medios o alternativa que se utilizan para el desarrollo de la personalidad de los escolares y elevar así el desempeño profesional pedagógico de los maestros, en cumplimiento con los objetivos planteados en los lineamientos aprobados en el VI Congreso del Partido Comunista de Cuba (PCC) con relación a la esfera educacional.

El concepto formación está implícito, en el pensamiento educativo martiano, en su aspiración de formar: "Hombres vivos, hombres directos, hombres independientes, hombres amantes, eso han de hacer las escuelas que ahora no hacen eso" . Al mismo tiempo proclamó que la educación debería propiciar la formación de un hombre nuevo: libre, integral, multifacético crítico, con cualidades morales elevadas, con opiniones propias.

En el campo de la pedagogía se han considerado los aportes de diferentes autores acerca de la categoría formación (G. Ferry, 1997; Bernard Honore, Lothelier, citados por Moreno, 2003; Vaillant, 2001 y otros). En general, en sus definiciones se valora la formación como un proceso, una función o una capacidad evolutiva, como una actividad que tiene como máxima pretensión el desarrollo de las potencialidades del individuo.

Se destaca en la formación la participación activa de los individuos, a partir de la interacción con otros y la posición reflexiva y autor reflexiva que asumen. Los análisis de diversos autores también permiten valorar la relación de la formación con los procesos de desarrollo y educación. La formación implica desarrollo y se logra a través de la educación.

La Comisión Internacional de la Educación para el siglo XXI (Informe Delors 1990) destaca el rol de los educadores, señalando que: "Los educadores para el próximo milenio necesitan formarse en un nuevo paradigma: el paradigma del aprendizaje, en el cual los educadores son primordialmente diseñadores de métodos y ambientes de aprendizaje, que trabajan en equipo junto a los estudiantes, de suerte que en realidad devienen en coaprendices. El educador, a la vez que forma, se está formando, y a la vez que

Martí, J. 1985:86. 
enseña aprende" (Tünnerman, 2003; p 11).

La UNESCO (1974) define formación permanente como un proceso dirigido a la revisión y renovación de conocimientos, actitudes y habilidades previamente adquiridas, determinado por la necesidad de actualizar los conocimientos como consecuencia de los cambios, avances de la tecnología y de las ciencias.

La educación permanente es considerada por el Ministerio de Educación Superior, una prioridad importante a partir de valorarse la necesidad de la continua superación de los profesionales de la educación, según el Reglamento de Educación de Postgrado (2014), como una de las direcciones principales de la Educación Superior en Cuba, y el nivel más alto de este subsistema, dirigido a promover la educación permanente de los graduados universitarios, en correspondencia con el desarrollo de la ciencia, la técnica y la tecnología, como contenidos fundamentales de la cultura general integral requeridos para la actuación competente de estos profesionales.

De esta forma se concuerda con el reglamento que la Educación de Postgrado, se estructura en formación académica y en superación profesional. La formación académica tiene como objetivo la educación posgraduada con una alta competencia profesional investigativa e innovadora. La superación profesional tiene como objetivo la formación permanente y la actualización sistemática de los graduados universitarios, el perfeccionamiento del desempeño de sus actividades profesionales y académicas, así como su acervo cultural.

En Cuba se identifican diferentes momentos históricos, en el desarrollo de la formación permanente del maestro primario, que se han desarrollado en las condiciones económicas, políticas y sociales en la que se ha perfeccionado el sistema educativo cubano, en correspondencias con las transformaciones desarrolladas en cada nivel de educación.

\section{Tendencia de la formación permanente del maestro primario en Cuba.}

Desde el triunfo de la Revolución se comienzan a dar pasos para instaurar y consolidar un sistema educativo que pueda enfrentar el reto de una educación masiva, de calidad y de participación social que involucra a las diferentes agencias sociales, todo lo que incide en el fortalecimiento de la escuela y en la formación del maestros, hubo un predominio de la actividad académica en la formación que impregnó la concepción pedagógica, que no favorecía la formación socio educativa del maestro, lo que limita el desempeño de sus roles profesionales en la solución de los problemas escolares como un aspecto fundamental de la época y no se habla de método, ni de conflictos escolares, se prioriza la formación académica del maestro.

La proyección en la formación permanente parte de problemáticas de carácter nacional, no se tenían en cuenta las necesidades de otros niveles organizativos de la sociedad (regiones, provincias), ni las dimensiones metodológicas e investigativa, por lo que los directivos no daban solución a los problemas técnicos y/o pedagógicos dada la aplicación de los elementos teórico- prácticos del trabajo metodológico 
y la investigación.

El proceso de formación permanente de los maestros solo abarca el uso de cursos emergentes como forma organizativa fundamental, cuestión que limita la capacidad para la adopción de estrategias amplias que perfeccionen la planificación, organización, ejecución y control.

El nivel de dirección fue centralizado desde el Ministerio de Educación, la mayoría de las acciones ejecutadas por el Instituto de Perfeccionamiento Educacional y derivadas hasta las escuelas, dirigidas a garantizar la formación pedagógica elemental básica para responder al derecho de todos los cubanos sin distinción de raza, color, sexo, clase, lugar donde vivían, a la educación.

La proyección de actividades de superación, dirigidas por los Institutos de Perfeccionamiento Educacional para atender las dificultades de los docentes, según su nivel de desarrollo, carece de verdaderos procesos, que integrados, permitiera configurar una dirección científica acorde a las necesidades del territorio y de los docentes.

A partir de 1975 hasta 1999 se produce el perfeccionamiento del personal docente de la educación. En esta etapa se revela que la formación permanente de los maestros primarios transita de una concepción espontánea, poco organizada y en ocasiones improvisada, a una concepción estructurada teniendo en cuenta las necesidades más generales, sin embargo quedan muchas demandas específicas que deben ser atendidas y resueltas sistemáticamente.

Las vías y acciones para la formación permanente de los maestros evolucionaron desde la centralización hacia la descentralización de la superación que permite responder mejor a las necesidades específicas de estos y a la solución de problemas concretos de las escuelas.

Las características de la formación permanente del maestro primario parten del reconocimiento del auto preparación, autodidactismo espontáneo, en las primeras décadas del proceso revolucionario y evolucionan hacia un esquema irregular signado por políticas que alternan de la centralización a la descentralización dirigidas a necesidades generales y ocasionalmente a aspectos específicos de su desempeño profesional.

A partir de 1999 hasta la fecha se puede afirmar que la formación permanente en el orden técnico, pedagógico, científico y metodológico obedece de forma concreta a la concentración, fundamentalmente, en las micro universidades creadas en los municipios de cada provincia, combinada con formas complementarias tales como: la implementación de Seminarios Nacionales y actividades dirigidas por los Institutos Superiores Pedagógicos.

La dirección contempla el predominio del método de trabajo independiente y del investigativo y mayor acercamiento a los contextos de actuación de los maestros.

La búsqueda de alternativas en la dirección de la superación profesional continua que permitan la atención permanente al desarrollo de las competencias requeridas para la profesión, aunque a pesar de 
los intentos realizados no se ha logrado totalmente el tratamiento de las necesidades formativas y de formación permanente que presentan los docentes, en especial de aquellos que se forman o ya han sido formados como profesores adjuntos.

La aprobación de la Resolución Ministerial 132/ 2004, Reglamento de la Educación de Postgrado que establece una nueva visión del proceso de dirección de la superación profesional y deroga la $\mathrm{R} / \mathrm{M}$ 6/96. Dentro de los cambios que se introducen, se encuentran la aprobación de la auto superación, la conferencia especializada, el taller y el debate científico como formas organizativas y la definición de los créditos que aportan los diferentes cursos de superación.

El empleo de modalidades fundamentales en la formación permanente tales como: Cursos cortos y el Diplomado, utilizadas como alternativas para otorgar titulaciones, con este fin se implementa un plan curricular único durante un período de tiempo prolongado, sin evidenciarse cambios significativos en la concepción curricular.

El estudio histórico permitió identificar que si bien los cambios responden a las transformaciones educacionales, no siempre la preparación de los maestros resulta suficiente para elevar la calidad de la educación, a lo que contribuyen insuficiencias en su formación, esto se evidencia con mayor notoriedad al aplicarse el modelo de universalización de la Educación Superior Pedagógica donde aparecen nuevos conceptos entre los que resultan significativos: tutor, docente en formación, micro universidad, profesor a tiempo parcial. Estos cambios requieren de elevadas exigencias a la labor del maestro, así como de su constante formación y autoformación.

\section{Concepción de la estrategia de formación permanente del maestro primario para la mediación en conflictos.}

Partimos de considerar que las estrategias, son concluidas como resultados científicos en la investigación educativa, desde una diversidad de interpretaciones, la palabra estrategia aparece con una frecuencia no des estimable en los estudios asociados al campo de la educación, donde se considera un resultado de significación práctica, ya que la misma tiene como propósito esencial la proyección del proceso de transformación del objeto de estudio desde un estado real hasta un estado deseado. (...) (De Armas Ramírez Nerely, Josefa Lorences González y José Manuel Perdomo Vázquez. Caracterización y diseño de los resultados científicos como aporte de la investigación educativa. Universidad de Ciencias Pedagógicas "Félix Varela". Villa Clara. Cuba 2008, pág. 36)

"La estrategia establece la dirección inteligente, y desde una perspectiva amplia y global, de las acciones encaminadas a resolver los problemas detectados en un determinado segmento de la actividad humana (...)”(A F, Fátima y otros, 1999: p. 26 y 27).

“(...) las contradicciones o discrepancias entre el estado actual y el deseado, entre lo que es y debería ser, de acuerdo con determinadas expectativas (...)" que dimanan de un proyecto social y/o educativo dado. 
Su diseño implica la articulación dialéctica entre los objetivos (metas perseguidas) y la metodología (vías instrumentadas para alcanzarlas). (G H, Alicia M. /y/ otros, 1998: p. 39-40)

"Entendemos por estrategia cierto ordenamiento de las acciones en el curso de la resolución de un problema en el cual cada paso es necesario para el siguiente. Estas secuencias de acciones están fuertemente orientadas hacia el fin a alcanzar.

Es una regularidad y así se puede constatar que las estrategias permiten proyectar un cambio cualitativo en el sistema, a partir de eliminar las contradicciones entre el estado actual y el deseado. En el ámbito pedagógico tiene entre sus direcciones fundamentales:

Es una forma de expresar la dirección de un proceso en la educación, que parte de objetivos, necesidades, intereses, proyectos, conocimientos y comportamiento de los sujetos.

Responde a un referente contextualizado, que consta de dos momentos imprescindibles: teórico e interventiva, donde los recursos humanos representan el núcleo del desarrollo.

Muestra la consistencia, la firmeza, estabilidad, coherencia, resistencia, solidez, duración del comportamiento esperado, al señalar las direcciones específicas en la eficiencia de los resultados en la educación.

Está compuesta por elementos prácticos (acciones), para que exista una adecuada conexión entre pensamiento-acción y de esta manera poder transformar eficientemente las realidades educativas.

El diagnóstico constituye su punto de partida, en tanto, revela tener en cuenta las potencialidades de los sujetos y procesos objeto del cambio.

La estrategia para la apropiación del método de mediación en conflictos escolares desde la formación permanente del maestro primario permite definir qué hacer para que los maestros primarios se apropien del método de mediación educativa en conflictos escolares, para lograr calidad en la labor educativa de la escuela primaria cubana.

\section{Entre los rasgos que distinguen la estrategia se encuentran:}

Carácter procesal y sistémico: dada que está estructurada en etapas y acciones, entre las cuales se establecen estrechas relaciones para conformar un todo dinámico, que evoluciona constantemente.

Enfoque interdisciplinar: establecido por el vínculo entre conocimientos, modos de actuación, valores, desde el punto de vista teórico - práctico y considerando el desempeño profesional pedagógico del maestro primario.

Flexibilidad: parte de considerar las características de los maestros a que va dirigida, de ahí la posibilidad de contextualizarse según las nuevas condiciones que favorezcan la aspiración de elevar el desempeño profesional pedagógico del maestro primario. 
Carácter transformador: proporciona transformaciones en el desempeño de la labor educativa del maestro primario.

Participativa, por que compromete en el proceso asumir una posición responsable en el desarrollo de las actividades, bien planificadas de manera que la participación sea de forma directa, permite crear, innovar, introducir nuevas propuestas y soluciones a los problemas que se van presentando, con precisión en las actividades que se proponen con coherencia.

Es humanista al considerar al maestro como centro de la atención en la transformación de su desempeño.

En ella se evidencia una postura reflexiva, a partir de la dinámica entre su esencia pedagógica y su aplicación.

Mantiene una visión perspectiva orientada hacia el futuro.

Adquiere una concepción contextualizada al responder a las necesidades y características de la realidad del maestro primario.

Además permite introducir las premisas para utilizar el método de mediación educativa por el maestro, con un enfoque sistémico en el que predominan las relaciones de coordinación, aunque no dejan de estar presentes las relaciones de subordinación y dependencia.

Una estructuración a partir de etapas relacionadas con las acciones de diagnóstico, planificación, aplicación y evaluación. El hecho de responder a una contradicción entre el estado actual y el deseado de un objeto concreto ubicado en el espacio y en el tiempo que se resuelve mediante la utilización programada de determinados recursos psicológicos y métodos educativos.

Tiene un carácter dialéctico que le viene dado por la búsqueda del cambio cualitativo que se producirá en el proceso de formación permanente del maestro primario (estado real ha estado deseado), por las constantes adecuaciones y readecuaciones que puede sufrir su accionar y por la articulación entre los objetivos (metas perseguidas) y la metodología (vías instrumentadas para alcanzarlas), entre otras.

Su carácter de aporte eminentemente práctico debido a sus persistentes grados de utilidad. Ello no niega la existencia de aportes teóricos dentro de su conformación.

\section{Exigencias metodológicas:}

- Considerar la superación como el espacio importante de formación permanente para el desarrollo profesional pedagógico del maestro primario.

- Implica la relación interdisciplinar con los fundamentos teóricos necesarios para comprender la labor del maestro y la calidad en su desempeño profesional. 


\section{Fundamentos teóricos de la estrategia.}

Esta estrategia se sustenta teóricamente en enfoque psicológico: De los psicólogos Dr. C. González Rey Fernando, Dr. C. Del Pino Jorge Luís, quienes sustentan la necesidad del conocimiento de la personalidad, en la formación multilateral de la misma como objetivo fundamental de nuestra sociedad y es el principio esencial de la educación, por lo que se debe tener en cuenta sus motivos e intereses, aspiraciones, desarrollo psíquico, físico, entre otros.

Esencialmente se adscribe al enfoque histórico cultural, de L. S. Vigostky, y su pensamiento psicológico, donde se destaca el papel de las relaciones interpersonales en la actividad humana, el significado de las vivencias para el desarrollo del sujeto y el principio que la enseñanza conduce al desarrollo, lo antecede y lo guía. Donde lo persono lógico se manifiesta por el respeto y formación de la personalidad.

Enfoque sociológico: Es asumido para el estudio de la influencia de la herencia biológica y del medio externo en el desarrollo psíquico e intelectual del escolar, y la existencia de diferentes etapas en su desarrollo evolutivo. El hombre es un ser social, cuyos aprendizajes, en su totalidad, de una manera u otra, están mediados por la influencia que sobre él ejerce el entorno en que se desenvuelve, por lo tanto sus éxitos o fracasos no dependen exclusivamente del aspecto individual, sino son resultado de las múltiples y diversas interacciones que establece con sus semejantes, lo biológico es solamente un aspecto. El ser vive en sociedad y tiene relaciones directa con el medio que lo rodea, estableciendo un enfrentamiento entre el medio natural y social a partir de la cooperación y comunicación, cuando esto ocurre la educación es fundamental.

Enfoque pedagógico: En la respuesta educativa personalizada a los maestros primarios para trazar las estrategias más adecuadas, que le permitan introducir oportunamente las premisas necesarias para lograr mediar en conflictos y lo conduzcan al éxito educativo, de acuerdo con las capacidades y necesidades de cada uno de ellos.

Enfoque persono lógico: Atendiendo a que es necesario tener en cuenta la interacción de los escolares entre sí y con el sistema de relaciones sociales en que se desarrollan, sus diferencias individuales, los motivos, necesidades, vivencias afectivas, el nivel de socialización para su transformación y progreso sistemático para transitar a etapas superiores.

Enfoque psicológico: atendiendo al conocimiento de las características psicológicas que se presenta en la edad escolar para propiciar un adecuado tratamiento a las manifestaciones propias de este periodo, comprenderlas y detectar cualquier dificultad que se pueda presentar.

Al asumir los postulados de la Escuela - Histórico- Cultural y el enfoque persono lógico como los referentes teóricos de esta investigación, tienen en cuenta los puntos de vista acerca del papel que la cultura y la historia personal desempeñan en la socialización de los individuos. De lo anterior se deduce que las dinámicas y cambiantes relaciones entre las personas, las cuales tienen diferentes formas y grados de manifestación, desde las más favorecedoras, hasta las que colocan al individuo en situación 
de desventaja, determinan su inserción en la cultura, con mayor o menor grado de ajuste y calidad en las respuestas que aporta a las demandas de su entorno.

El aprendizaje específicamente humano, está subordinado, en gran medida, a la Ley Genética Fundamental del Desarrollo, por lo tanto tiene un primer plano ubicado en el mundo de los interacciones humanas, como una función interpsíquica, que obliga a tener en cuenta como se produce la interiorización, en relación con la riqueza de las experiencias sociales que se trasmiten al individuo, por lo tanto no es único responsable de los saberes socialmente determinados.

Ley dinámica del desarrollo o situación social del desarrollo: Cada momento del desarrollo se caracteriza por una peculiar combinación de factores internos y externos, que condiciona las vivencias que tienen el sujeto en ese período y los nuevos logros o desarrollos psicológicos de la etapa. Se establece pues una compleja y particular dialéctica que incluye lo biológico, las adquisiciones ya formadas y toda la gama de influencias significativas en un momento histórico concreto de la sociedad y la vida de cada sujeto.

De lo anterior se deduce que el nivel de relación individual está mediado por las características del ambiente en que tiene lugar y de lo favorecedor que resulte el mismo para la interiorización de los distintos aprendizajes, a sí mismo explica como una dificultad no puede ser vista solamente a partir de la identificación de los déficit individuales, al contrario, exige se tomen en cuenta todos los elementos que se implican en el proceso.

Las posibilidades de aprendizaje de modos de comportamiento no se determinan únicamente, a partir de lo que un sujeto en un momento determinado haga o de lo que es capaz de resolver, sino que contempla lo que puede llegar a ser si la respuesta a sus demandas es ajustada.

La comprensión del papel de la cultura en el desarrollo de las Funciones Psíquicas Superiores resulta esencial para entender las razones que permitan afirmar que las dificultades en el área de la socialización, no pueden interpretarse únicamente poniendo la mirada en la situación particular del escolar, en el aspecto del desarrollo biológico y que necesariamente tiene que involucrarse el medio social en el que tiene lugar el desarrollo.

\section{Etapas de la Estrategia.}

\section{I. - Fase de Diagnóstico.}

Objetivo: Diagnosticar la preparación de los maestros primarios sobre los conocimientos básicos de tres elementos fundamentales: los conflictos escolares, (el conocimiento de condiciones, causas, manifestaciones y factores que lo provocan), así como lo resuelven, como realizan la intervención educativa, qué método utilizan en su desempeño profesional educativo y qué vías utiliza para su formación permanente en esta área de su desempeño. (Ver anexos 1,2 y 3) y mediante la observación a clases, la entrevista y encuesta a los maestros. 
Se analizó las características profesionales de los maestros en las dimensiones de: años de experiencias, categorías académicas y científicas y tiempo de trabajo en el ciclo, por lo que se determinó (a la muestra seleccionada) sus principales necesidades de preparación localizadas en: lo teórico metodológico e investigativo.

\section{Fase de Planificación}

Objetivo: Determinar los temas a desarrollar en función de las necesidades de formación de los maestros en correspondencia con el diagnóstico desarrollado.

En cada aspecto de preparación se trazarán objetivos específicos, contenidos a desarrollar y las formas de actividad. El beneficiario directo es el maestro primario, el cual tendrá la oportunidad de ir elevando su preparación profesional pedagógica. El beneficiario indirecto será, en lo esencial, el escolar. No obstante, también contribuye a estimular las buenas actitudes y comportamientos de los agentes socializadores de la comunidad (familia, instituciones culturales y educacionales).

Preparación coherente y lógica de los talleres en función de tres elementos fundamentales: los conflictos escolares, (el conocimiento de condiciones, causas, manifestaciones y factores que lo provocan), así como lo resuelven, como realizan la intervención educativa, qué método utilizan en su desempeño profesional educativo y qué vías utiliza para su formación permanente en esta área de su desempeño.

\section{Acciones a desarrollar:}

Elaborar diagnóstico según indicadores. (Conocimiento de las manifestaciones de los conflictos escolares, causas, factores que lo determinan, métodos para resolver conflictos escolares y vía de superación que recibe en el centro)

Aplicar diagnóstico. Procesar diagnóstico. Determinar las dificultades y potencialidades. Instrumentar o establecer los niveles de socialización con los directivos de la unidad docente seleccionada. Preparar a los maestros primarios sobre la definición de conflictos escolares, sus causas, condiciones, factores y manifestaciones en el contexto educativo para ello se socializaron los procedimientos a utilizar para el estudio general sobre conflictos y mediación desde el entrenamiento psicopedagógico, como una vía de formación permanente y sensibilizó al claustro hacia el conocimiento de los componentes psicológicos, pedagógicos y sociológicos de la naturaleza de los conflictos escolares y las premisas, condiciones y factores necesarios para apropiarse del método de mediación en conflictos escolares.

\section{Fase de Planificación:}

Se realiza con el objetivo de determinar los temas a desarrollar en función de las necesidades de formación de los maestros en correspondencia con el diagnóstico desarrollado.

Preparación coherente y lógica de los talleres en función de tres elementos fundamentales antes mencionados. 


\section{Acciones a desarrollar:}

Seleccionar las formas y tipos del trabajo metodológico. Las formas son:

- Docente- metodológica que tiene como tipos: preparación de la carrera, de las disciplinas, de las asignaturas, reunión metodológica,_clase metodológica, clase abierta, de comprobación y taller metodológico.

- Científico- metodológica tiene entre sus tipos: trabajo científico- metodológico del maestro y de los colectivos metodológicos, seminarios científico- metodológicos y conferencias científicometodológicas.

- Seleccionar la forma, tiempo de duración y espacio dentro del sistema de trabajo de la entidad docente

- Organizar un postgrado en forma de entrenamiento. Coordinar con la DME, DPE y la dirección de postgrado de la UCP Frank País García. Coordinar con los implicados la matrícula.

\section{Fase de Ejecución:}

Se realiza con el objetivo de propiciar la introducción de actividades metodológicas en las que se manifiesten las relaciones entre las manifestaciones, causas y factores en que se manifiestan los conflictos escolares y las premisas y condiciones necesarias para apropiarse del método de mediación educativa como una vía de formación permanente del maestro primario para elevar su desempeño profesional.

Impartir los talleres en las actividades metodológicas que realiza la escuela previa coordinación con el centro.

Se realiza la preparación a los maestros desde la preparación metodológica en la propia escuela con un tiempo de duración de un semestre, por 2 horas mensuales, a través de las modalidades del trabajo metodológico seleccionadas: auto preparación como forma individual del trabajo metodológico, talleres y seminarios como formas colectivas de este. En su conjunto tienen un carácter dialéctico, humanista e integrador y su propósito fundamental es lograr que el maestro primario se apropie del método de mediación educativa en conflictos escolares.

La auto preparación posibilita desarrollar y profundizar en los conocimientos referentes a la temática abordada en relación con el desempaño profesional pedagógico del maestro. Los seminarios científico metodológicos permiten resolver tareas dirigidas al desarrollo de hábitos y habilidades, profundizar en determinadas materias y apropiarse o sistematizar una metodología científica de estudio.

Los talleres metodológicos permiten promover discusiones y análisis colectivos sobre manifestaciones, causas y factores que originan los conflictos y sobre las premisas, condiciones y factores necesarios para apropiarse del método de mediación en conflictos escolares, a partir de la implementación del entrenamiento socio pedagógico (ver anexo 
La preparación metodológica permite fortalecer el desarrollo de la creatividad, elevar el nivel de preparación y desempeño profesional y se dirige, en este caso, a la aprehensión del método de mediación y a su incorporación en el proceso pedagógico.

\section{IV- Fase de evaluación y control.}

Se realiza con el objetivo de comprobar el desempeño profesional pedagógico de los maestros al resolver conflictos escolares en la escuela.

Esta etapa permite controlar y evaluar la pertinencia y efectividad de la estrategia aplicada como retroalimentación de las fases iníciales de la misma.

Esta estrategia se llevó a cabo en la escuela primaria Nguyen Van Troi, ubicada en el reparto sueño, de la provincia de Santiago de Cuba, de régimen sami interno, se trabajó con una muestra de 20 maestros, de ellos 14 maestros del segundo ciclo (5to y 6to grado) y 6 especialistas.

Para el diagnóstico inicial se realizaron encuestas, entrevistas a maestros, directivos y especialistas, así como la revisión de documentos. Se estimó como indicador para el diagnóstico inicial, los tipos y contenidos de la superación del territorio o del centro. Para determinar los conocimientos básicos de tres elementos fundamentales: los conflictos escolares, (el conocimiento de condiciones, causas, manifestaciones y factores que lo provocan), así como lo resuelven, como realizan la intervención educativa, qué método utilizan en su desempeño profesional educativo y qué vías utiliza para su formación permanente en esta área de su desempeño.

Las orientaciones metodológicas generales para el desarrollo de las acciones de la estrategia para la apropiación del método de mediación en conflictos escolares desde la formación permanente del maestro primario, parten desde el enfoque dialéctico materialista e histórico de nuestra concepción pedagógica, lo que permitió darle tratamiento a determinados contenidos que favorecieron enriquecer los puntos de vistas teórico metodológicos y mayor cientificidad en el análisis de los mismos.

Se propone realizar un entrenamiento socio pedagógico con la muestra seleccionada resultado del diagnóstico, con el objetivo de que los maestros se apropien del método a través del control y evaluación de las acciones previstas en la estrategia en sus tres etapas.

Alos efectos prácticos de la presente investigación la estrategia se implementó de forma parcial, al abarcar solamente a la muestra seleccionada intencionalmente y a partir de los propios resultados obtenidos en el diagnóstico inicial. Se desarrolló a través del trabajo metodológico, reflejándose en el cumplimiento de las acciones propuestas en cuanto a las modalidades del auto preparación, talleres y seminarios. Las mismas contribuyeron a profundizar en las dimensiones teórico metodológico e investigativa referentes al conocimiento de los conflictos y los fundamentos teóricos prácticos del método de mediación en conflictos y propiciaron niveles cualitativamente superiores en el comportamiento y actuación del maestro primario. 


\section{Resultados}

Los resultados concretos obtenidos se reflejan de la siguiente forma, según los indicadores empleados se aprecia una evolución cuantitativa en cuanto al conocimiento del método mediación educativa en conflictos ascendente a 20 maestros que manifiestan estar mejores preparados y capacitados para enfrentar los retos y desafíos que se presentan en la práctica pedagógica y social al poseer un método coherente y profundo para identificar y resolver conflictos.

Se obtiene un aumento en catorce maestros con relación al dominio de las condiciones, factores y manifestaciones del conflicto, permitiéndoles actuar con conocimiento de causa en el sentido de identificar los indicadores de estabilidad, frecuencia e intensidad con que se presentan situaciones de conflictos en los mismos escolares. En su conjunto aprendieron a ser mejores personas y a influir con mayor eficiencia y eficacia en los escolares, la familia y la comunidad.

Sin lugar a dudas, el aporte más importante de los resultados parciales obtenidos, es que los maestros objetos de transformación comprobaron que el trabajo metodológico es y tiene que convertirse en el contexto fundamental para su superación, preparación y formación permanente.

Sus valoraciones concluyen al expresar la necesidad de su aplicación generalizada en la escuela en los dos ciclos porque propicia mayores fortalezas en el desarrollo del sistema de trabajo preventivo educativo y se convierte en dinamizador del mismo.

La aplicabilidad de la propuesta desde el trabajo metodológico, denota su importancia como elemento esencial integrador en el desarrollo del proceso docente educativo y la necesidad de su incremento en todos los espacios del contexto educativo.

\section{CONCLUSIÓN}

La elevación de la calidad de la educación, como consecuencia de los niveles de desarrollo alcanzados en nuestro país, ha demandado el perfeccionamiento continuo de la formación de maestros, de modo que sean capaces de enfrentar de manera creadora la solución permanente de los problemas que se le presentan en su desempeño profesional.

La mediación es un método para resolver conflictos con un alto potencial educativo, que demanda ser utilizado por el maestro para mejorar las relaciones interpersonales en el grupo, favorecen el ambiente escolar y el aprendizaje de los mismos, así como la formación de valores ciudadanos y el desempeño profesional en la dirección del proceso educativo. 


\section{REFERENCIAS BIBLIOGRÁFICAS}

Addine, F. (2004). Didáctica: teoría y práctica. Editorial Pueblo y Educación, Ciudad de la Habana, Cuba.

Álvarez de Zayas, C. (1999). La escuela en la vida. Editorial Pueblo y Educación, La Habana, Cuba.

Añorga, J., Pérez, A. y Valcárcel, N. (1996). Las formas de la Educación Avanzada: hacia una propuesta integral (Material impreso). Instituto Superior Pedagógico "Enrique José Varona”; La Habana: 42.

Beltrán, J. (1987): Estrategias de aprendizaje. En BELTRÁN, J. y otros: Psicología de la educación. Eudema. Madrid.

Beltrán, J. (2002). Las claves psicológicas de la convivencia escolar. Ponencia. Seminario Convivencia en los centros escolares como factor de calidad, Madrid.

Castañedo, A. (1999), Mediación, alternativa para la resolución de conflictos, La Habana, Ediciones ONBC.

Cherly, A. (2002). Mediación en conflictos interpersonales y de pequeños grupos. Publicaciones Acuario. Centro Félix Varela.

Colectivo de autores. (2002). Reflexiones teórico prácticas desde las Ciencias de la Educación. La Habana.

Comenius, J. (1983). Didáctica Magna. Editorial Pueblo y Educación, La Habana, Cuba.

Cortina, A. (1997).Resolver conflictos hacer justicia, Cuaderno de Pedagogía № 257, España, Abril

Davidov, A. (1988). La enseñanza escolar y el desarrollo psíquico. Editorial Progreso, Moscú, Cuba.

Engativá y Kennedy (2007): Gestión del conflicto escolar. Ministerio Federal de corporación economía y desarrollo, Bogotá, Colombia. Recuperado de: http://www.paho.org/CDMEDIA/FCHGTZ/ INFORMACION\%20DE\%20LOS\%20PAISES/COLOMBIA/sistematizaciones/Texto4/Gestion\%20 del\%20conflicto\%20escolar.pdfrevisado22/02/2012

Entelman, R. (2001). Teoría de conflictos. Hacia un nuevo paradigma. Barcelona: Gedisa

Fuentes, M. (2001). Mediación en la solución de conflictos: Publicación Acuario. Centro Félix Varela. La Habana P 21- 53

Hernández, de la Requera, José Antonio (2007). Conflicto, Comunicación y Liderazgo Escolar: Los vértices de un triángulo equilátero. Profesorado. Revista de Curriculum y formación del profesorado. Universidad de Granada. http://www.ugr.es/local/recfpro/rev113 COL3.pdfrevisado 22/02/2012

Hernández, P, María Ángeles (2000).Los conflictos en el aula. http// www.definición.org/comunidad/ 
aprendizaje $02 / 2013$

Ibarra, L. (s.f) .Educar en la Escuela, educar en la familia, ¿realidad o utopía? La Habana: Editorial Félix Varela.

Ibarra, L. (2007). Los Conflictos Escolares: Un problema de todos. Facultad de Psicología - Universidad de la Habana. Recuperado de: http://www.psicologiaonline.com/articulos/2007/conflictos_escolares. shtmlrevisado 8/04/2012

Jarres, X. (1994). "El lugar del conflicto en la organización escolar". En Revista Iberoamericana de Educación, $\mathrm{N}^{\mathrm{o}} 15, \mathrm{p} .54$.

Kolb, D. M.819859, « To be a Mediator: Expressive Tactics in Mediation », Journal of Social Issues, 41 (2): $11-27$

López, J. (2000). Fundamentos de la Educación. Editorial Pueblo y Educación, Ciudad de la Habana.

Ortega Rosario y del Rey Rosario (2003): Estrategias educativas para la prevención de la violencia. Mediación o diálogo. Edita: Cruz Roja Juventud. Departamento Central c/Rafael Villa, s/n El Plantio.28023 Madrid- p,18

Pedagógicas. ISP Félix Varela. Villa Clara. Ruiz Aguilera, Ariel. (2003) La Investigación Educativa. Material digital del IPLAC.

Rico, P. (2000). Hacia el perfeccionamiento de la escuela primaria. Editorial Pueblo y Educación, Ciudad de la Habana, 2000.

Rodríguez del Castillo, M. (2002). "Estrategia metodológica para la preparación postgraduada de los docentes en la lectura de la obra martiana”. Tesis en opción al título de Doctora en Ciencias

Vigotsky, L. (1987) Historia del Desarrollo de las Funciones Psíquicas Superiores, Editorial Científico Técnica, Ciudad de la Habana, Cuba. 